Review

\title{
Management of mechanical valve thrombosis during pregnancy, case report and review of the literature
}

\author{
Çağdaş Akgüllü *, Ufuk Eryılmaz, Hasan Güngör, Cemil Zencir \\ Adnan Menderes University Faculty of Medicine, Cardiology Department, Turkey
}

\section{A R T I C L E I N F O}

\section{Article history:}

Received 7 September 2017

Received in revised form 13 September 2017

Accepted 13 September 2017

Available online 14 September 2017

\begin{abstract}
A B S T R A C T
Anticoagulant therapy of the patients with mechanical heart valve prosthesis (MHV) in the course of pregnancy requires careful monitorization and well estimation of each step regarding benefits and handicaps of each treatment strategy in the particular trimester. Unfractioned heparin with close monitoring of activated thromboplastin time (APTT), low molecular weight heparin with close monitoring of anti Xa levels or warfarin with close monitoring of INR are the main options. It may be challenging most of the sometimes because of the procoagulant nature of pregnancy as well as physiological changes like increased glomerular filtration rate. During the follow up, any recent onset symptom should call prompt and careful investigation beginning with transthoracic echocardiography and planning further transesophageal echocardiography and fluoroscopic studies if needed. If MHV thrombosis is detected, management of patients differs due to the presence of obstruction, critical illness, thromboembolic events or thrombus size. Thrombolytic therapy and the surgical thrombectomy are the options for critically ill patients. International guidelines suggest surgical approach as a first line therapy if the risk of surgery is not too high. However, the complication and success rates of studies with fibrinolytic agents are encouraging. Each strategy comes with its own particular risk and regardless of the selected strategy MHV thrombosis during the pregnancy is a high risk situation. In this paper, we report a 26 year old patient presented with recent onset dyspnea due to MHV thrombosis in the mitral position. After the failure of unfractioned heparin, and because of hemodynamic deterioration she was referred for urgent surgery. She recovered after the surgery, however baby was found to have congenital diaphragmatic hernia and is still monitored in the intensive care unit. This report includes, treatment strategies of anticoagulant medication for the pregnant patients with MHV prosthesis and management of MHV thrombosis during the pregnancy.
\end{abstract}

(C) 2017 The Society of Cardiovascular Academy. Production and hosting by Elsevier B.V. All rights reserved. This is an open access article under the CC BY-NC-ND license (http://creativecommons.org/licenses/by-nc-nd/4.0/).

\section{Contents}

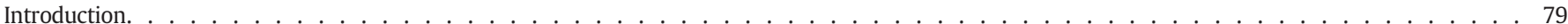

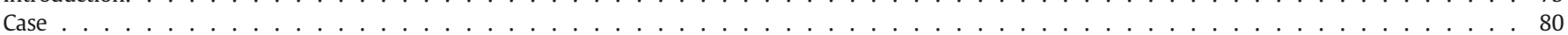

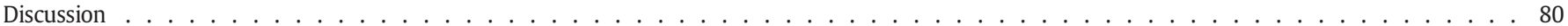

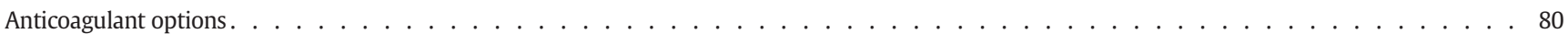

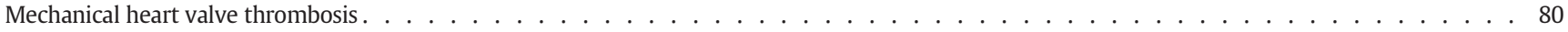

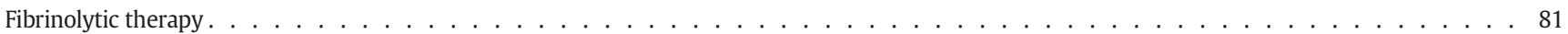

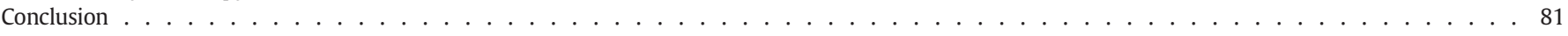

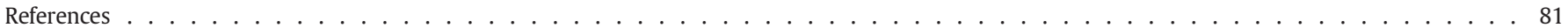

\footnotetext{
* Corresponding author at: Adnan Menderes University Faculty of Medicine, Cardiology Department, Aytepe Mevkii, Efeler, Aydın, Turkey.

E-mail address: cagdasakgullu@gmail.com (Ç. Akgüllü).

Peer review under responsibility of The Society of Cardiovascular Academy.
}

\section{Introduction}

Mechanical valve thrombosis during pregnancy is a life threatening situation. Accurate treatment is unclear and management should be personalized to find the lowest acceptable risk both for the fetus and the mother. Each strategy has its own particular risk and potential 
benefit. Clinicians should estimate the risk of each step carefully on this blurry road while trying to reach final cure for their patients.

\section{Case}

Twenty six year old woman at her 36 week of pregnancy presented to our emergency department with dyspnea and fatigue. The patient had a history of mechanical mitral valve implantation because of rheumatic disease in 2012. Transthoracic echocardiography (TTE) showed prosthetic valve dysfunction with high transvalvular gradient (max: $30 \mathrm{~mm} \mathrm{Hg}$, mean $18 \mathrm{~mm} \mathrm{Hg}$ ) without significant regurgitation. Pulmonary artery systolic pressure was calculated to be $68 \mathrm{~mm} \mathrm{Hg}$ (from the tricuspid valve velocity). She was taking subcutaneous $80 \mathrm{mg}$ (equivalent to 8000 IU anti-Xa activity) enoxaparin two times a day and was taking no other anticoagulant medications. Her anti Xa levels was measured and found to be in therapeutic range $(1.3 \mathrm{IU} / \mathrm{ml})$. However, transesophageal echocardiography (TEE) revealed thrombus on the atrial side to cause restriction of leaflet motions (Fig. 1). Intravenous continuous infusion of unfractionated heparin (UFH) to maintain activated partial thromboplastin time (APTT) 1,5 to 2 times higher than the upper normal threshold of controls was begun. However urgent surgical consultation was made in the second day due to deteriorating clinical status. She was also consulted to the gynecology department. The decision to take baby via cesarean section and to continue with the extirpation of thrombus in the same operation session was made. Just after the delivery, the baby was resuscitated and intubated and found to have right pulmonary hypoplasia and congenital diaphragmatic hernia. After the extraction of thrombus, mother was okay and extubated in the second day of operation. She was discharged in the tenth day with $100 \mathrm{mg}$ acetyl salicylic acid (ASA) and adjusted warfarin, while baby was still under monitorization in the intensive care unit.

\section{Discussion}

\section{Anticoagulant options}

Management of the anticoagulant regimen of women with mechanical heart valves (MHV) during pregnancy is a challenging problem because of the procoagulant nature of pregnancy. Especially in the second and third trimesters, increased levels of factors VII, VIII, and X and decreased levels of protein $S$ lead to a hypercoagulable state. ${ }^{1}$ On the other hand thrombotic complications of MHV mostly occur during the first trimester where anticoagulant regimens are switched from one

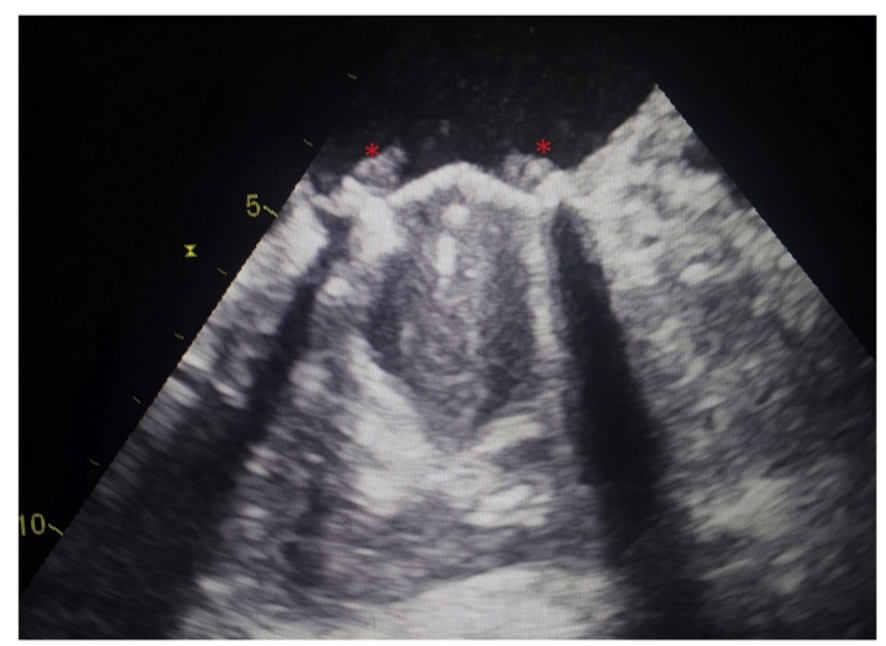

Fig. 1. Transesophageal echocardiography image depicting diffuse thrombus particles on the atrial side of mitral mechanic prosthetic valve. (Red asterisk on the both sides). regimen to another. ${ }^{2}$ Oral vitamin $\mathrm{K}$ antagonists (VKA) are the most effective regimen proven to prevent thrombosis of MHVs. However, VKA are shown to cross placental barrier and cause a group of embryonal pathology known as warfarin embryopathy., ${ }^{3,4}$ These include nasal hypoplasia and stippled epiphyses, ocular and neurological abnormalities. VKA was also shown to cause late fetal loss and stillbirth. ${ }^{5}$ The risk of embryopathy is dose dependent, with a low risk $(<3 \%)$ if the dose of warfarin is $\leq 5 \mathrm{mg}$ per day. ${ }^{5}$ Moreover in the first trimester, the risk of fetal loss and abortion is same in the course of low dose warfarin, UFH or low molecular weight heparin (LMWH) usage. ${ }^{5,6}$ Thus, if $\leq 5 \mathrm{mg}$ warfarin dose can maintain therapeutic INR levels, it may be possible not to switch the anticoagulant regimen to another option. But if higher doses are needed to achieve therapeutic levels, then switching the therapy to another anticoagulant regimen other than VKA is needed. At that point the risk of valve thrombosis and related complications begins to rise. It is possible to use subcutaneous UFH throughout the pregnancy not to meet any embryonal pathology. However this strategy was shown to be related to high incidence (up to 33\%) of MHV thrombosis. ${ }^{7}$ Current guideline of the American College of Cardiology/American Heart Association (ACC/AHA) valvular heart disease guidelines suggests that if therapeutic targets of INR is achieved with $<5 \mathrm{mg}$ /day warfarin usage, then it should be used throughout pregnancy. ${ }^{5,6}$ However, if the dose to achieve therapeutic INR exceeds $5 \mathrm{mg}$ /day, changing the anticoagulant regimen from warfarin to LMWH or continuous UFH during the first trimester is recommended. ${ }^{5,6}$ After substitution to a VKA, it is recommended until 36 weeks, when it should be replaced with LMWH or UFH mainly to prevent fetal intracranial hemorrhage as well as uncontrolled bleeding of the mother during the vaginal delivery. Doseadjusted continuous intravenous UFH (with an aPTT at least 2 times control) during the first trimester is suggested for pregnant patients with a mechanical prosthesis if the dose of warfarin exceeds $5 \mathrm{mg}$ /day to achieve a therapeutic INR. ${ }^{5}$ On the other hand, if LMWH is the preferred agent in the first trimester, it is recommended to adjust the dosage not depending on weight but to maintain peak anti-Xa levels between 1.0 and $1.2 \mathrm{U} / \mathrm{ml}$ that was checked after $4-6 \mathrm{~h}$ of the last dose because of the fact that dose requirements may increase by as much as $50 \%$ over the course of pregnancy especially due to increased glomerular filtration rates. ${ }^{5,8}$ LMWH provides better bioavailability, longer half-life, and a more predictable anticoagulation response, and it does not cross placental barrier. ${ }^{5}$ It is known that monitoring anti-Xa levels in patients under LMWH regimen reduces complication rates as it was reported that maternal thromboembolic events were $22 \%$ versus $8.64 \%$ and death was $4 \%$ versus $0 \%$ with and without monitoring. ${ }^{9-11}$ Moreover, with use of this anti-Xa dependent dosing regimen, the incidence of valve thrombosis is lower than with UFH. ${ }^{5}$ On the other hand, it was shown that despite appropriate anti-Xa levels, MHV thrombosis risk is still a problem in patients under management of LMWHs.

\section{Mechanical heart valve thrombosis}

It is usually hard to decide about MHV thrombosis during the pregnancy as cardiac output increases throughout the pregnancy which leads to limited increment of mean pressure gradient across the prosthetic valves. However, MHV thrombosis should be promptly suspected in cases presented with recent onset dyspnea and/or embolic events. ${ }^{12}$ First TTE and then, if suspicion is high TEE should be performed. ${ }^{13}$ If it is still required, fluoroscopy may be performed with limited fetal risk. ${ }^{13}$ In a review, MHV thrombosis rates were reported to be $3.9 \%$ when oral anticoagulants (OACs) are selected throughout the pregnancy and 9.2\% when UFH is used in the first and OACs are used in the second and third trimesters. ${ }^{7}$ The rate is high as $33 \%$ when UFH is selected to be used throughout all trimesters. ${ }^{7}$

MHV thrombosis is a high risk situation, and this is regardless of the selected management strategy. Fibrinolytic therapy comes with the risks of bleeding and embolic events and surgery carries high maternal and fetal risks as it will be held in emergency situation and is a 
reintervention. ${ }^{13,14}$ Recurrent thrombosis rate is higher when fibrinolytic therapy is chosen. ${ }^{14}$ The management of MHV thrombosis is similar to the non-pregnant patients. On the other hand, there may be some differences by means of fetal risk. Recently published "2017 ESC/EACTS Guidelines for the management of valvular heart disease" guideline suggests different management algorithms for the obstructive and non-obstructive MHV thrombosis. ${ }^{14}$ For the obstructive MHV thrombosis, if the patient is critically ill then emergent surgery is suggested when there is no contraindication to the operation and immediate access is possible. However, if patient is not so critical then one must check the situation of recent adequate anticoagulation. If the answer is no then following under UFH $+100 \mathrm{mg}$ daily ASA is suggested. If there is no success with that therapy then surgery is suggested if there is not any high surgical risk. On the other hand, if patient is presented with obstructive MHV thrombosis and not critically ill and is under adequate anticoagulation, then direct surgical approach is suggested if the surgical risk is not too high. If surgical risk is high, fibrinolytic therapy is suggested instead. $^{14}$

If the presentation of MHV thrombosis is non obstructive, then the status of embolic events should be questioned. If there is clinically or documented embolism (with imaging technologies) and thrombus was smaller than $10 \mathrm{~mm}$, optimization of anticoagulation and close monitoring is advised. If it won't resolve or the size won't decrease and if it doesn't cause any recurrent thromboembolic event (TE), just close follow up is suggested. On the other hand, if recurrent TE occurs, then surgery or if the surgical risk is high, fibrinolytic therapy is suggested. If there is clinically or documented embolism (with imaging technologies) and thrombus was bigger than $10 \mathrm{~mm}$, then surgery or if the surgical risk is high, fibrinolytic therapy is suggested. ${ }^{14}$

When MHV thrombosis is non obstructive and there is not any TE and the size of the thrombus is bigger than $10 \mathrm{~mm}$, then optimization of anticoagulant therapy and close follow up is suggested. But, if thrombus persists or TE occurs during follow up, then surgery or if the surgical risk is high, fibrinolytic therapy is suggested. When the size is smaller than $10 \mathrm{~mm}$, follow up is suggested if the thrombus disappears, its size decreases, or it persists without TE after optimization of the anticoagulant therapy. However, if it causes TE, then surgery or if the surgical risk is high, fibrinolytic therapy is suggested. ${ }^{14}$

To prevent further TE, optimization of risk factors and close monitoring of the anticoagulation is suggested. Moreover, the addition of lowdose aspirin ( $\leq 100 \mathrm{mg}$ daily) after careful analysis of the risk-benefit ratio, is advised. ${ }^{14}$

\section{Fibrinolytic therapy}

Though the risk of surgical approach is very high, the particular risks of the fibrinolytic therapy limits its first line utilization in cases of MHV thrombosis in pregnant patients. Fibrinolytic agents cannot cross the placenta but may cause severe bleeding and subplacental haematoma. Moreover, the risk of embolization is high as $10 \%$, and the appropriate dose of fibrinolytic agents during pregnancy is still controversial as the experience is limited. Nevertheless, fibrinolysis may be preferred in right-sided prosthetic valve thrombosis after the information of the family about the risks. Recent ESC guide suggests fibrinolysis (using recombinant tissue plasminogen activator $10 \mathrm{mg}$ bolus $+90 \mathrm{mg}$ in $90 \mathrm{~min}$ with UFH or streptokinase $1,500,000 \mathrm{U}$ in $60 \mathrm{~min}$ without UFH) with class 2a suggestion, when surgery is not available or is very high risk or for thrombosis of right-sided prostheses. ${ }^{14}$ There is not any specific fibrinolytic dose suggestion for the pregnant patients, the guideline suggests using the same dose. On the other hand, Özkan et al. demonstrated that repeated doses of low-dose $(25 \mathrm{mg}$ ) and slow infusion (6 h) alteplase under the guidance of serial TEE was superior to faster infusion and/or higher dose protocols or streptokinase. ${ }^{15}$ They also demonstrated the effectiveness of this regimen on pregnant patients with MHV thrombosis. ${ }^{16}$ Moreover, recently same group demonstrated that ultra-slow (25 h) infusion of low-dose (25 mg) alteplase without bolus appears to be associated with quite low complications and mortality for MHV thrombosis patients without making any sacrifice from the success. ${ }^{17}$ They reported the success of that regimen to be $90 \% .{ }^{17} \mathrm{Re}-$ cent meta-analysis of Castelho et al. reported that mortality in patients treated by thrombolytic therapy for MHV thrombosis is significantly lower than in patients treated surgically. ${ }^{18}$ It is known that fetal loss is very high with surgery, thus fibrinolysis may be considered instead of surgery in non-critically ill patients when anticoagulation fails. ${ }^{19}$

\section{Conclusion}

Prevention of MHV thrombosis in the pregnancy begins with the first contact of patients in the first trimester. This requires full assessment of the characteristics of patients and then to decide the right anticoagulant regimen for each particular patient. During the follow up, factor anti Xa or INR levels should be monitored closely as needed. Each new onset symptom needs full re-examination. If MHV thrombosis is detected it should be known that it is a very high risk situation both for the fetus and the mother and that high risk is independent from the management strategy. Guidelines offer surgical approach as a first line therapy for the critically ill patients with obstructive MHV thrombosis. On the other hand, fibrinolysis is still an option for the selected patients. Further randomised studies comparing the outcomes of surgery versus pregnancy adjusted doses of fibrinolytic agents may change future management suggestions.

\section{References}

1. James AH. Pregnancy-associated thrombosis. Hematology Am Soc Hematol Educ Program 2009:277-285.

2. Van Hagen IM, Roos-Hesselink JW, Ruys TP, et al, for the ROPAC Investigators and the EURObservational Research Programme (EORP) Team. Pregnancy in women with a mechanical heart valve: data of the European Society of Cardiology Registry of Pregnancy and Cardiac Disease (ROPAC). Circulation 2015;132: $132-142$.

3. Hall JG, Pauli RM, Wilson KM. Maternal and fetal sequelae of anticoagulation during pregnancy. Am J Med 1980;68:122-140.

4. McLintock C. Anticoagulant choices in pregnant women with mechanical heart valves: balancing maternal and fetal risks-the difference the dose makes. Thromb Res 2013;131(suppl 1):S8-10.

5. Nishimura RA, Otto CM, Bonow RO, et al. 2014 AHA/ACC guideline for the management of patients with valvular heart disease: executive summary: a report of the American College of Cardiology/American Heart Association Task Force on Practice Guidelines. J Am Coll Cardiol 2014;63:2438-2488.

6. Xu Z, Fan J, Luo X, et al. Anticoagulation regimens during pregnancy in patients with mechanical heart valves: a systematic review and meta-analysis. Can J Cardiol 2016 Oct;32(10), https://doi.org/10.1016/j.cjca.2015 (1248.e1-1248.e9).

7. Chan WS, Anand S, Ginsberg JS. Anticoagulation of pregnant women with mechanical heart valves: a systematic review of the literature. Arch Intern Med 2000;160: 191-196.

8. Alshawabkeh L, Economy KE, Valente AM. Anticoagulation during pregnancy: evolving strategies with a focus on mechanical valves. J Am Coll Cardiol 2016 Oct 18;68(16):1804-1813.

9. James AH, Brancazio LR, Gehrig TR, et al. Low molecular-weight heparin for thromboprophylaxis in pregnant women with mechanical heart valves. J Matern Fetal Neonatal Med 2006;19:543-549.

10. Oran B, Lee-Parritz A, Ansell J. Low molecular weight heparin for the prophylaxis of thromboembolism in women with prosthetic mechanical heart valves during pregnancy. Thromb Haemost 2004;92:747-751.

11. Suri V, Keepanasseril A, Aggarwal N, et al. Mechanical valve prosthesis and anticoagulation regimens in pregnancy: a tertiary centre experience. Eur J Obstet Gynecol Reprod Biol 2011 Dec;159(2):320-323.

12. Karthikeyan G, Senguttuvan NB, Joseph J, Devasenapathy N, Bahl VK, Airan B. Urgent surgery compared with fibrinolytic therapy for the treatment of left-sided prosthetic heart valve thrombosis: a systematic review and meta-analysis of observational studies. Eur Heart J 2013;34:1557-1566.

13. European Society of Gynecology, Association for European Paediatric Cardiology, German Society for Gender Medicine, et al. ESC guidelines on the management of cardiovascular diseases during pregnancy: the Task Force on the Management of Cardiovascular Diseases during Pregnancy of the European Society of Cardiology. Eur Heart J 2011;32:3147-3197.

14. The Task Force for the Management of Valvular Heart Disease of the European Society of Cardiology (ESC), the European Association for Cardio-Thoracic Surgery (EACTS). 2017 ESC/EACTS guidelines for the management of valvular heart disease. Eur Heart J 2017;00:1-53. 
15. Özkan M, Çakal B, Karakoyun S, et al. Thrombolytic therapy for the treatment of prosthetic heart valve thrombosis in pregnancy with low-dose, slow infusion of tissue-type plasminogen activator. Circulation 2013;128:532-540.

16. Özkan M, Gündüz S, Biteker M, et al. Comparison of different TEE-guided thrombolytic regimens for prosthetic valve thrombosis: the TROIA trial. JACC Cardiovasc Imaging 2013 Feb;6(2):206-216.

17. Özkan M, Gündüz S, Gürsoy OM, et al. Ultraslow thrombolytic therapy: a novel strategy in the management of PROsthetic MEchanical valve Thrombosis and the prEdictors of outcomE: the ultra-slow PROMETEE trial. Am Heart J 2015 Jun;12.
18. Castilho FM, De Sousa MR, Mendonça AL, Ribeiro AL, Cáceres-Lóriga FM. Thrombolytic therapy or surgery for valve prosthesis thrombosis: systematic review and meta-analysis. J Thromb Haemost 2014 Aug;12(8):1218-1228.

19. Weiss BM, von Segesser LK, Alon E, Seifert B, Turina MI. Outcome of cardiovascular surgery and pregnancy: a systematic review of the period 1984-1996. Am J Obstet Gynecol 1998;179:1643-1653. 